\section{TALKING POINTS 2014 - BOOK NOW}

After almost 30 years of excellence in

learning, the Talking Points in Dentistry

lecture series is back again in 2014,

promising to bring you interesting and

relevant sessions with practical learnings

you can take back to your practice, all

brought to you by renowned experts in

the field.

This year, due to popular demand, GSK have added a new location to the line-up.

Cardiff, and will visit a total of 11 venues

throughout the UK. Also, for the

first time, the lecture series has

been split by both date and

topic, allowing Talking Points to

cover more of the topical issues

in dentistry; six events will take

place in May, with a further five

in September.

MAY: Professor David Bartlett and clinical psychologist,

Professor Tim Newton from King's

College London present new

thinking on the condition of acid

wear. With one in three young

adults' affected by acid wear,

they will provide a unique insight

into how you can influence

\section{patient behaviours to prevent further}

damage.

SEPTEMBER: Dates and information to be announced soon!

Don't miss out! The limited places are always booked up quickly for this exciting lecture series. Contact your GSK representative or visit www. gskdentalprofessionals.co.uk

. Bartlett D W, Lussi A, West N X, Bouchard P, Sanz $M$, Bourgeois D. Prevalence of tooth wear on buccal and lingual surfaces and possible risk factors in young European adults. J Dent 2013:41: 1007-1013

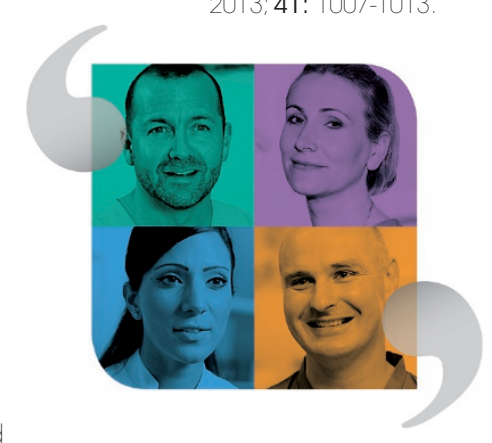

\section{Talking Points in Dentistry}

TOP TEN TIPS FOR PARENTS

From Philips, creators of the

newly-launched latest version of Sonicare for Kids (pictured).

1. Brush your child's teeth for them until the age of seven

2. Children should consume fizzy drinks, smoothies and juice as a treat. preferably sticking to milk and water at other times

3. If children do consume fizzy drinks, smoothies and juice, they should be encouraged to do so through a straw

4. Restrict sweets, fruit juices, smoothies and fizzy drinks to meal times

5. Drink water afterwards to wash away the acid

6. Do not brush the teeth until an hour after eating or drinking

7. Wean children off drinking milk from bottles by the age of one as soon as possible after they start eating proper food and certainly by the age of 18-24 months

8. Children should not be allowed to take a bottle or feeder cup to bed

9. Drinking juices from a feeder cup should be avoided

10. Use fluoridated toothpaste twice daily.

\title{
EARLY ACID WEAR SCREENING ESSENTIAL
}

Acid wear is a leading cause of tooth wear. Results from the pan-European ESCARCEL study reveal that almost one in three young adults have experienced tooth wear and more than two in five demonstrate dentine hypersensitivity, a related condition.

Previous research by GSK found that most dental professionals think that acid wear is becoming more common. ${ }^{2}$ Now, the results from the first pan-European Epidemiology Study on Non-Carious Cervical Lesions (ESCARCEL) show just how common acid wear has become. A particularly worrying insight is the prevalence of the condition amongst young adults - the study found that almost a third of the European young adult population suffers from tooth wear. $29.4 \%$ of $18-25$-year-olds suffered from tooth wear, with an even higher prevalence in 26-35-year-olds. The study found more severe tooth wear in those who frequently consumed acidic foods and drinks.

\section{PRONAMEL}

The study also found that $41.9 \%$ of young adults suffer from dentine hypersensitivity, a condition which is often associated with tooth wear. Indeed, the study's findings confirm a strong association between erosive factors (such as high consumption of dietary acids, or gastric reflux) and dentine hypersensitivity, an association which previous studies, which hadn't assessed both conditions simultaneously, have been unable to prove.

ESCARCEL shows that an acidic diet leads to higher levels of tooth wear. Simple advice like avoiding acidic foods and drinks between mealtimes could help your patients avoid acid wear. You can also recommend a toothpaste designed specifically to help protect against the effects of acid wear, like Pronamel Daily Toothpaste. Pronamel is proven to help reharden acid-softened enamel and build protection against future acid challenges. ${ }^{3}$

1. West NX, Sanz M, Lussi A, Bartlett D, Bouchard P. Bourgeois D. Prevalence of dentine hypersensitivity and study of associated factors: a European population-based crosssectional study. J Dent 2013; 41: 847-851.

2. GSK Data on File 2013.

3. Hara AT, Kelly S A, González C et al. Influence of fluoride availability of dentifrices on eroded enamel remineralization in situ. Caries Res 2008; 43: 57-63. 